\title{
THE MODIFIED ENSKOG EQUATION FOR MIXTURES
}

\author{
H. VAN BEIJEREN \\ Instituut voor Theoretische Fysica, Katholieke Universiteit Nijmegen, \\ Nijmegen, Nederland
}

M.H. ERNST

Instituut voor Theoretische Fysica, Rijksuniversiteit Utrecht, Utrecht, Nederland

Received 21 June 1973

\section{Synopsis}

In a previous paper it was shown that a modified form of the Enskog equation, applied to mixtures of hard spheres, should be considered as the correct extension of the usual Enskog equation to the case of mixtures. The main argument was that the modified Enskog equation leads to linear transport coefficients which are in complete agreement with the laws of irreversible thermodynamics. The existing extensions of the Enskog equation to the case of mixtures, on the other hand, yield results which do not satisfy the Onsager reciprocity relations. In this paper the detailed derivation of these results is presented, i.e., the normal solution of the modified Enskog equation for mixtures is obtained, the Navier-Stokes equations are derived and the explicit expressions for the linear transport coefficients which follow, are shown to satisfy the laws of irreversible thermodynamics.

1. Introduction. In a recent paper ${ }^{1}$ ), referred to as $I$, we have proposed a modified Enskog equation for a dense gas of hard spheres. As has been discussed in the literature ${ }^{2}$ ) the existing extensions of the usual Enskog equation to the case of hard-sphere mixtures ${ }^{3,4,5}$ ) do not reproduce the linear relations of irreversible thermodynamics ${ }^{6}$ ) between fluxes and conjugate driving forces in a correct way; e.g., the Onsager reciprocity relations are in general not satisfied.

In this paper we shall show that the modified Enskog equation for an $s$-component mixture of hard spheres of diameter $\sigma_{i}$ and mass $m_{i}(i=1,2, \ldots, s)$ leads directly to linear relations between fluxes and conjugate driving forces, which are in complete agreement with the laws of irreversible thermodynamics. In addition we shall explicitly evaluate the linear transport coefficients. To start with we shall review in section 1 some of the results which were obtained in I. We give the linearized form of the modified Enskog equation in its Fourier-transformed re- 
presentation and repeat the definitions of the relevant quantities. In section 2 the linearized equation is solved to linear order in the Fourier variable $k$, i.e., to terms linear in the gradients, with the aid of the projection-operator formalism. Then by taking the long-time limit the so-called normal solution is obtained in which the single-particle distribution function (D.F.) depends on time through the hydrodynamic variables only.

In section 3 the Navier-Stokes equations are derived. First the time derivatives of the hydrodynamic variables are expressed in the usual way as divergences of corresponding fluxes. Next, using the results of section 2, we show that these fluxes are proportional to the conjugate driving forces, in accordance with the laws of irreversible thermodynamics. The proportionality coefficients are identified as linear transport coefficients, for which we obtain explicit expressions. From the properties of the Enskog collision operator we deduce that the matrix of transport coefficients is positive definite and symmetric, which implies that the Onsager reciprocity relations are satisfied.

Now let us consider the modified Enskog equation. Since we are only interested in the linear transport coefficients we find it convenient to work with its linearized form, derived in $\mathrm{I}$, which reads

$$
\frac{\partial}{\partial t} h=-L h
$$

where

$$
L=L(k)=K(k)+D(k)+\Lambda(k),
$$

with

$$
\begin{aligned}
(K h)_{i}= & \mathrm{i} k \cdot v_{t} h_{i}, \\
(D h)_{i}= & -\mathrm{i} k \cdot v_{i} \sum_{j}\left[\tilde{C}_{i j}(k)-\chi_{i j} \tilde{f}_{i j}(k)\right] \int \mathrm{d} v_{j} \varphi_{j} h_{j}, \\
(\Lambda h)_{i}= & -\sum_{J} \chi_{i j} \int \mathrm{d} v_{j} \varphi_{j} \sigma_{i j}^{2} \int^{\prime} \mathrm{d} \hat{\sigma}\left(v_{i j} \cdot \hat{\sigma}\right) \\
& \times\left(h_{i}^{*}-h_{i}+\mathrm{e}^{-\mathrm{i} k \cdot \hat{\sigma} \sigma_{l j}} h_{j}^{*}-\mathrm{e}^{\mathrm{i} k \cdot \hat{\sigma} \sigma_{l j}} h_{j}\right) .
\end{aligned}
$$

The function $h_{i}$ is the Fourier transform of the relative deviation $\varphi_{i}^{-1}\left(F_{i}-\varphi_{i}\right)$ of the single-particle D.F., $F_{l}$, from its equilibrium value $\varphi_{i}$. This function is the Maxwell-Boltzmann D.F.,

$$
\varphi_{i}=n_{i}\left(\beta m_{i} / 2 \pi\right)^{3 / 2} \exp \left(-\frac{1}{2} \beta m_{i} v_{i}^{2}\right),
$$

where $n_{t}$ is the partial density of species $i$ in thermal equilibrium, $T=\left(k_{\mathrm{B}} \beta\right)^{-1}$ is the equilibrium temperature and $k_{\mathrm{B}}$ is Boltzmann's constant. The D.F. $h$ depends 
on the time $t$, the Fourier variable $k$, the discrete variable $i$ labelling the different species, and the continuous variable $\boldsymbol{v}_{\boldsymbol{i}}$, denoting the velocity of a particle belonging to species $i$. We shall in general use the short-hand notations $(h)_{t}=h_{i} \equiv h_{i}\left(v_{i}, k, t\right)$ and in a similar way $(k h)_{i} e t c$. Furthermore $h_{i}^{*} \equiv h_{i}\left(v_{i}^{*}, k, t\right)$ and similarly for $h_{j}^{*}$, where the velocities $v_{i}^{*}$ and $v_{j}^{*}$ of the restituting collision are given in terms of the velocities $v_{i}$ and $v_{j}$ of the direct collision

$$
\boldsymbol{v}_{i}^{*}=\boldsymbol{v}_{i}-2 \frac{\mu_{t j}}{m_{i}}\left(v_{i j} \cdot \hat{\sigma}\right) \hat{\sigma}, \quad v_{j}^{*}=v_{j}+2 \frac{\mu_{t j}}{m_{j}}\left(v_{i j} \cdot \hat{\sigma}\right) \hat{\sigma}
$$

Here $v_{i j}=v_{i}-v_{j}$, the reduced mass $\mu_{i j}$ is defined by $\mu_{t j}^{-1}=m_{i}^{-1}+m_{j}^{-1}$. Occasionally we shall also use $M_{i j}=m_{i}+m_{j}$. A caret on a symbol (e.g., $\left.\hat{\sigma}\right)$ denotes a unit vector. The integration over the solid angle $d \hat{\sigma}$ in $(1.2 c)$ is restricted to the hemisphere $v_{i j} \cdot \hat{\sigma}>0$, which is indicated by a prime on the integral sign. We further have $\chi_{i j}=g_{i j}\left(\sigma_{i j}\right)$ where $g_{i j}(r)$ is the equilibrium radial D.F. for two particles belonging to species $i$ and $j$ and $\sigma_{i j}=\frac{1}{2}\left(\sigma_{i}+\sigma_{J}\right)$. Further, $\tilde{f}_{i j}(k)$ is the Fourier transform of the Mayer function $f_{i j}\left(r_{i j}\right)$ (which equals -1 for $r_{i j}<\sigma_{i j}$ and vanishes elsewhere), given by

$$
\tilde{f}_{i j}(k)=\int \mathrm{d} \boldsymbol{r} \mathrm{e}^{-\mathrm{i} k \cdot \dot{r}} f_{i j}(r)=\left(4 \pi \sigma_{i j}^{2} / k\right) j_{1}\left(k \sigma_{i j}\right), \quad \tilde{f}_{i j}(0)=-\frac{4}{3} \pi \sigma_{i j}^{3},
$$

where $j_{i}(x)=x^{-1} \cos x-x^{-2} \sin x$ is a spherical Bessel function. Similarly $C_{i j}(k)$ is the Fourier transform of the direct correlation function, defined by the OrnsteinZernike relation

$$
\tilde{G}_{i j}(k)=\widetilde{C}_{i j}(k)+\sum_{l} n_{l} \tilde{C}_{l l}(k) G_{l j}(k),
$$

where $\tilde{G}_{i j}(k)$ is the Fourier transform of the pair correlation function $G_{i j}(r)$ $=g_{t j}(r)-1$. Once the distribution function is known, one can calculate average values $\delta \psi=\delta \psi(k, t)$ of the deviations from equilibrium of some microscopic function $\psi_{i}\left(v_{i}\right)$ by means of the relation,

$$
\delta \psi=(\psi, h),
$$

where we have introduced the inner product

$$
(\psi, h)=\sum_{i} \int \mathrm{d} v_{i} \varphi_{i} \psi_{i} h_{i}
$$

In particular we shall frequently consider the set of microscopic functions $\left\{\psi_{a}\right\}$, referred to as summational invariants or hydrodynamic variables, i.e.,

$$
\psi_{a}=\left\{\psi_{l}, \psi_{u}, \psi_{T}\right\} \quad(l=1,2, \ldots, s),
$$


where

$$
\psi_{l i}=\delta_{l i} ; \quad \psi_{u i}=m_{i} v_{i} ; \quad \psi_{T i}=\frac{1}{2} m_{i} v_{i}^{2}-\frac{3}{2} \beta^{-1}
$$

and their averages

$$
\delta n_{l}=\left(\psi_{l}, h\right), \quad \varrho \delta \boldsymbol{u}=\left(\psi_{u}, h\right), \quad \frac{3}{2} n k_{\mathbf{B}} \delta T=\left(\psi_{T}, h\right) .
$$

Here $\delta n_{l}$ is the Fourier transform of the deviation of the local partial density from its equilibrium value; $\delta u$ the Fourier transform of the local mass velocity and $\delta T$ the Fourier transform of the deviation of the local temperature from its equilibrium value. We further introduced the total mass density $\varrho=\sum_{l} \varrho_{i}=\sum_{i} m_{t} n_{i}$ and the total number density $n=\sum_{i} n_{i}$.

In the sequel we shall also need the transposed of the operators (1.2), defined by the relation

$$
(\psi, L \xi)=\left(\xi, L^{T} \psi\right)
$$

where $\psi$ and $\xi$ are arbitrary functions of $i$ and $v_{i}$. Similar relations define $K^{T}, D^{T}$ and $A^{T}$. Their explicit forms can be obtained directly from (1.2) and read:

$$
\begin{aligned}
\left(K^{T} h\right)_{i}= & (K h)_{i}=i k \cdot v_{i} h_{i}, \\
\left(D^{T} h\right)_{i}= & -\sum_{j}\left[\tilde{C}_{i j}(k)-\chi_{i j} \tilde{f}_{i j}(k)\right] \int \mathrm{d} v_{j} \varphi_{j} \mathrm{i} k \cdot v_{j} h_{j}, \\
\left(\Lambda^{T} h\right)_{i}= & -\sum_{j} \chi_{i j} \int \mathrm{d} v_{j} \varphi_{j} \sigma_{i j}^{2} \\
& \times \int^{\prime} \mathrm{d} \hat{\sigma}\left(v_{i j} \cdot \hat{\sigma}\right)\left[h_{i}^{*}-h_{i}+\mathrm{e}^{-\mathrm{i} k \cdot \hat{\sigma} \sigma_{i j}}\left(h_{j}^{*}-h\right)\right] .
\end{aligned}
$$

From $(1.2 \mathrm{c}),(1.12 \mathrm{c})$ and the relation

$$
\sigma_{i j}^{2} \int^{\prime} \mathrm{d} \hat{\sigma} \hat{\sigma}\left(\mathrm{e}^{-\mathrm{i} k \cdot \hat{\sigma} \sigma_{l j}}-\mathrm{e}^{\mathrm{i} k \cdot \hat{\sigma} \sigma_{l j}}\right)=\int \mathrm{d} r \mathrm{e}^{-\mathrm{i} k \cdot r} \frac{\partial f_{i j}(r)}{\partial r},
$$

one can show that

$$
\Lambda=\Lambda^{T}+\Delta
$$

with

$$
(\Delta h)_{i}=-\sum_{j} \chi_{i j} f_{i j}(k) \int \mathrm{d} v_{j} \varphi_{j} \mathrm{i} k \cdot v_{i j} h_{j} .
$$

In view of the small $k$ behaviour, to be studied in the next section, we consider the limiting value of $\Lambda(k)$ for small $k$, i.e.,

$$
(\Lambda(0) \psi)_{i}=-\sum_{j} \chi_{i j} \int \mathrm{d} v_{j} \varphi_{j} \sigma_{i j}^{2} \int^{\prime} \mathrm{d} \hat{\sigma}\left(\boldsymbol{v}_{i j} \cdot \hat{\sigma}\right)\left(\psi_{i}^{*}+\psi_{j}^{*}-\psi_{i}-\psi_{j}\right),
$$


where $\psi_{i}$ is an arbitrary function of $i$ and $v_{i}$. The operator $\Lambda(0)$ is symmetric, i.e.,

$$
\Lambda^{T}(0)=\Lambda(0),
$$

and non-negative, i.e.,

$$
(\psi, \Lambda(0) \psi) \geq 0 \text {. }
$$

The only functions for which the equality sign in (1.17) holds are the summational invariants (1.9), which satisfy the relations

$$
\Lambda(0) \psi_{a}=\Lambda^{T}(0) \psi_{a}=0
$$

2. Solution of the linearized Enskog equation. The usual method for solving the kinetic equation (1.1) is the Chapman-Enskog method. The basic idea of the method is that for long times the time dependence of the D.F. $h$, is completely determined by the local hydrodynamical variables, defined in (1.10), i.e., the local partial densities $\delta n_{l}(t)$, the local mass velocity $\delta u(t)$, and the local temperature $\delta T(t)$. A more modern variant of the above procedure is the projection-operator method, introduced by $Z_{\text {wanzig }}$ ). This method employs the basic idea of the Chapman-Enskog procedure by introducing a projection operator $\mathscr{P}$, which projects microscopic functions of the variables $\left(i, v_{i}\right)$ on the hydrodynamic subspace, spanned by the hydrodynamic variables $\left\{\psi_{a}\right\}$, defined in (1.9). The connection between the two methods has been discussed in the literature $\left.{ }^{8}\right)$.

In the projection-operator method one determines equations of motion for the functions $P h$ and $P_{\perp} h$, with

$$
h=P h+P_{\perp} h \quad\left(P_{\perp}=1-P\right),
$$

by multiplying (1.1) by $P$ and $P_{\perp}$, respectively, yielding

$$
\begin{aligned}
& \frac{\partial}{\partial t} P h=-P L P h-P L P_{\perp} h, \\
& \frac{\partial}{\partial t} P_{\perp} h=-P_{\perp} L P_{\perp} h-P_{\perp} L P h,
\end{aligned}
$$

where the last equation has the formal solution

$$
P_{\perp} h(t)=\mathrm{e}^{-t P_{\perp} L P_{\perp}} P_{\perp} h(0)-\int_{0}^{t} \mathrm{~d} \tau \mathrm{e}^{-\tau P_{\perp} L P_{\perp}} P_{\perp} L P h(t-\tau) .
$$

This solution is to be inserted in (2.2a) to obtain a closed equation for $P$. This equation will yield the hydrodynamic equations, whereas (2.3) approaches for long times the normal solution of the Chapman-Enskog method $^{9}$ ). 
The projection operator acting on an arbitrary microscopic function $\xi$ is defined as

$$
\begin{aligned}
P \xi & =\sum_{a} \psi_{a}\left(\psi_{a}, \xi\right) /\left(\psi_{a}, \psi_{a}\right) \\
& =\sum_{l} \psi_{l}\left(\psi_{l}, \xi\right) \frac{1}{n_{l}}+\psi_{u} \cdot\left(\psi_{u}, \xi\right) \frac{\beta}{\varrho}+\psi_{T}\left(\psi_{T}, \xi\right) \frac{2 \beta^{2}}{3 n},
\end{aligned}
$$

where the first sum runs over the set of functions (1.9) and the second sum runs over all $s$ components in the mixture. We further used the inner products

$$
\left(\psi_{l}, \psi_{l}\right)=n_{l} ; \quad\left(\psi_{u}^{\alpha}, \psi_{u}^{\beta}\right)=\frac{\varrho}{\beta} \delta_{\alpha \beta} ; \quad\left(\psi_{T}, \psi_{T}\right)=\frac{3}{2} \frac{n}{\beta^{2}}
$$

Greek superscripts $(\alpha, \beta, \ldots=x, y, z)$ refer to carte ian components of vectors and tensors, and we use Einstein's summation convention for repeated indices. Notice also that $\left(\psi_{a}, \psi_{b}\right)=0$ for $a \neq b$.

From (2.4) and (1.10) we find

$$
P h=\sum_{l} \psi_{l}\left(\delta n_{l} / n_{l}\right)+\beta \boldsymbol{\psi}_{u} \cdot \delta \boldsymbol{u}+\beta \psi_{T}(\delta T / T)
$$

which is precisely the linearized single-particle D.F. in local equilibrium.

One verifies directly that $P$ satisfies the requirements $P^{2}=P$, and $P_{\perp}^{2}=P_{\perp}$. This property is equivalent with the usual requirements

$$
\left(\psi_{a}, P h\right)=\left(\psi_{a}, h\right) \quad \text { or } \quad\left(\psi_{a}, P_{\perp} h\right)=0,
$$

stating that the local-equilibrium D.F. determines at any time completely the local partial densities, the local mass velocity and the local temperature.

We want to derive the hydrodynamic equations together with explicit expressions for the transport coefficients. In order to do so we have to evaluate the quantities in (2.2) and (2.3) to the required order in the Fourier variable $k$. The terms in (2.2a) linear in $k$ determine the Euler equations; the quadratic terms the Navier-Stokes equations; the cubic terms the Burnett equations and so on. In this paper we restrict ourselves to the Navier-Stokes equations. A method for obtaining the linear Burnett equations is treated in ref. 10.

We start this programme by evaluating the quantities $P L P, P_{\perp} L P$, and $P L P_{\perp}$ or its transpose $P_{\perp} L^{T} P$, appearing in (2.2) and (2.3). The quantity $P L P$ can be evaluated in closed form in terms of spherical Bessel functions. This is done in an appendix. For our purpose it suffices to have $P L P$ to terms of $\mathcal{O}\left(k^{2}\right)$, which are also given in the appendix. Here we simply quote the results to the relevant order 
in $k$ by giving the matrix elements $\left(\psi_{a}, L \psi_{b}\right)$ of $P L P$;

$$
\left(\psi_{a}, L \psi_{b}\right)=\left(\begin{array}{ccc}
0 & \mathrm{i} k \beta^{-1} n_{l} & 0 \\
\mathrm{i} k \beta^{-1} \sum_{i} n_{i} \Delta_{i l} & k^{2} \beta^{-1} \eta^{\prime}(1+2 \hat{k} \hat{k}) & \mathrm{i} k \beta^{-1} p \\
0 & \mathrm{i} k \beta^{-1} p & k^{2} \beta^{-1} \lambda^{\prime}
\end{array}\right) .
$$

The first $s$ rows and columns are labelled by $\psi_{l}(l=1,2, \ldots, s)$; the next three rows and columns by $\psi_{u}$ or $\psi_{u}^{\alpha}(\alpha=x, y, z)$, and the last row and column by $\psi_{T}$. Here 1 is the unit tensor and $p$ the equilibrium pressure of the hard-sphere mixture, given by

$$
\beta p=n-\frac{1}{2} \sum_{i j} n_{i} n_{j} \chi_{i j} \tilde{f}_{i j}(0) .
$$

We further have

$$
\Delta_{i j}=\delta_{i j}-n_{j} \tilde{C}_{i j}(0) \text {. }
$$

The quantities $\eta^{\prime}$ and $\lambda^{\prime}$ are some of the collisional transfer contributions to the viscosity and heat-conduction coefficient, respectively. Their values are given as

$$
\begin{aligned}
& \eta^{\prime}=\frac{4}{15} \sum_{i j} n_{i} n_{j} \chi_{i j} \sigma_{i j}^{4}\left(2 \pi \mu_{i j} / \beta\right)^{\frac{1}{2}}, \\
& \lambda^{\prime}=\frac{4}{3} \beta^{-1} \sum_{i j} n_{i} n_{j} \chi_{i j} \sigma_{i j}^{4}\left(2 \pi \mu_{i j} \beta\right)^{\frac{1}{2}}\left(M_{i j}^{-1}\right) .
\end{aligned}
$$

Next we have to evaluate $P_{\perp} L P=L P-P L P$ and $P_{\perp} L^{T} P=L^{T} P-P L^{T} P$ to $\mathcal{O}(k)$, appearing in (2.2a), (2.2b) and (2.3). The quantities $P L P$ and $P L^{T} P$ are already known from (2.8), since according to (1.11)

$$
\left(\psi_{a}, L^{T} \psi_{b}\right)=\left(\psi_{b}, L \psi_{a}\right)
$$

So we start by evaluating to $\mathcal{O}(k)$ the quantities $L P$ and $L^{T} P$, or their components $L \psi_{a}$ and $L^{T} \psi_{a}$. This can be done directly by using (1.2), (1.12) and (1.14) and expanding all terms to linear order in $k$. Notice that $\tilde{C}_{i j}(k)$ and $\tilde{f}_{i j}(k)$ contain only even powers of $k$, since $C_{i j}(r)$ and $f_{i j}(r)$ depend only on the magnitude of the vector $r$. The calculations are straightforward and similar to those in the appendix. The results to $\mathcal{O}(k)$ are

$$
\begin{aligned}
& \left(L \psi_{l}\right)_{i}=\mathrm{i} k \cdot v_{i} \Delta_{i l}, \\
& \left(L^{T} \psi_{l}\right)_{i}=\mathrm{i} k \cdot v_{i} \delta_{i l}, \\
& \left(L \psi_{u}\right)_{i}=\mathrm{i} k \beta^{-1}+\mathrm{i} k \cdot\left\{m_{i} v_{i} v_{i}\right\}_{s} A_{i}+\mathrm{i} k\left(\frac{1}{3} m_{i} v_{i}^{2}-\beta^{-1}\right) B_{i}, \\
& \left(L^{T} \psi_{u}\right)_{i}=\mathrm{i} k \beta^{-1} \sum_{i} \Delta_{i l}+\mathrm{i} k \cdot\left\{m_{i} v_{t} v_{i}\right\}_{s} A_{i}+\mathrm{i} k\left(\frac{1}{3} m_{i} v_{i}^{2}-\beta^{-1}\right) B_{i}, \\
& \left(L \psi_{T}\right)_{i}=\left(L^{T} \psi_{T}\right)_{i}=\mathrm{i} k \cdot v_{i}\left(\frac{1}{2} m_{i} v_{i}^{2}-\frac{5}{2} \beta^{-1}\right) E_{i}+\mathrm{i} k \cdot v_{i} \beta^{-1} F_{i},
\end{aligned}
$$


where $\left\{t_{\alpha \beta}\right\}_{s} \equiv t_{\alpha \beta}-\frac{1}{3} \delta_{\alpha \beta} t_{\gamma \gamma}$ indicates a traceless tensor, and

$$
\begin{aligned}
& A_{i}=1-\frac{2}{5} \sum_{j} n_{j} \chi_{i j} \tilde{f}_{i j}(0)\left(\mu_{i j} / m_{i}\right), \\
& B_{i}=1-\sum_{j} n_{j} \chi_{i j} \tilde{f}_{i j}(0)\left(\mu_{i j} / m_{i}\right), \\
& E_{i}=1-\frac{6}{5} \sum_{j} n_{j} \chi_{i j} f_{i j}(0)\left(\mu_{i j} / M_{i j}\right), \\
& F_{i}=1-\sum_{j} n_{j} \chi_{i j} f_{i j}(0)\left(\mu_{i j} / m_{j}\right) .
\end{aligned}
$$

Finally we evaluate $P_{\perp} L P$ and $P_{\perp} L^{T} P$, which read in component form

$$
P_{\perp} L \psi_{a}=L \psi_{a}-\sum_{b} \psi_{b}\left(\psi_{b}, L \psi_{a}\right)\left(\psi_{b}, \psi_{b}\right)^{-1}
$$

and a similar expression for $P_{\perp} L^{T} P$. Their explicit forms can be written down immediately, with the aid of (2.13), (2.8), (2.12) and (2.5):

$$
\begin{aligned}
\left(P_{\perp} L \psi_{l}\right)_{l}= & \mathrm{i} k \cdot m_{i} v_{i} \sum_{j}\left(\delta_{i j}-\varrho_{j} / \varrho\right) \Delta_{J l} / m_{j}, \\
\left(P_{\perp} L^{T} \psi_{l}\right)_{i}= & \mathrm{i} k \cdot m_{i} v_{i}\left(\delta_{i l}-\varrho_{l} l \varrho\right)\left(1 / m_{l}\right), \\
\left(P_{\perp} L \psi_{u}\right)_{t}= & \left(P_{\perp} L^{T} \psi_{u}\right)_{i}=\mathrm{i} k \cdot\left\{m_{i} v_{i} v_{i}\right\}_{\mathrm{S}} A_{i} \\
& +\mathrm{i} k\left(\frac{1}{3} m_{i} v_{i}^{2}-\beta^{-1}\right) \sum_{j}\left(\delta_{i j}-n_{j} / n\right) B_{j}, \\
\left(P_{\perp} L \psi_{T}\right)_{i}= & \left(P_{\perp} L^{T} \psi_{T}\right)_{i}=\mathrm{i} k \cdot v_{i}\left(\frac{1}{2} m_{i} v_{i}^{2}-\frac{5}{2} \beta^{-1}\right) E_{i} \\
& +\mathrm{i} k \cdot m_{i} v_{i} \beta^{-1} \sum_{j}\left(\delta_{i j}-\varrho_{j} / \varrho\right)\left(F_{j} / m_{j}\right) .
\end{aligned}
$$

For a complete $k$ expansion of (2.2) and (2.3) it is also necessary to determine $\exp \left(-t P_{\perp} L P_{\perp}\right)$ and $P h(t-\tau)$ in (2.3) to zeroth order in $k$. Since $\lim _{k \rightarrow 0} L(k)$ $=\Lambda(0)$, and since $P_{\perp} \Lambda(0) P_{\perp}=\Lambda(0)$, [i.e., the summational invariants $\psi_{a}$ are right and left eigenfunctions with zero eigenvalue, as can be seen from (1.18)] we can replace $\exp \left(-t P_{\perp} L P_{\perp}\right)$ by $\exp [-t \Lambda(0)]$. Furthermore, we expand $P h(t-\tau)=P h(t)+\tau(\partial / \partial t) P h(t)+\cdots$. The second term on the right can be neglected since it is of $\mathscr{O}(k)$. This follows from (2.2a), where $P L P$ is at least of $\mathcal{O}(k)$.

The operator $\exp \left(-t P_{\perp} L P_{\perp}\right)$, or its long-wavelength limit, $\exp [-t \Lambda(0)]$ in (2.3), acts on functions which are orthogonal to the null eigenfunctions of $A(0)$. Since the first nonvanishing eigenvalue $\omega_{0}$ of $\Lambda(0)$ is typically of order $\omega_{0} \approx n \chi^{\sigma^{2}} \bar{v}$ ( $\sigma$ is a typical hard-sphere diameter of the order of $\sigma_{i j} ; \bar{v}$ is an average velocity; and $\chi$ is of the order of $\chi_{i j}$ ) the first term in (2.3) vanishes for times $t$ much longer than the mean free time $\approx \omega_{0}^{-1}$; and in the same long-wavelength limit we may 
extend the upper limit on the time integral in (2.3) towards infinity, yielding for long times

$$
P_{\perp} h(t) \approx-\int_{0}^{\infty} \mathrm{d} \tau \mathrm{e}^{-\tau \Lambda(0)} P_{\perp} L P h(t)=-\Lambda^{-1}(0) P_{\perp} L P h(t) .
$$

This equation, together with $P h$, given in (2.6), is the so-called normal solution of the Enskog equation, which depends on time only through the time dependence of the local hydrodynamic variables $\delta n_{l}(t), \delta u(t)$ and $\delta T(t)$. The right-hand side of (2.17) is actually the formal solution of the Chapman-Enskog equations, which determines those parts of the single-particle D.F. which are linear in $k$, i.e., linear in the gradients of the local partial densities, the local mass velocity and the local temperature.

After completion of the $k$ expansion and the explicit evaluation of all quantities to the relevant order in $k$, we can derive the Navier-Stokes equations, and obtain expressions for the fluxes of mass, momentum and energy. This will be done in the next section.

3. Navier-Stokes equations. In this section we derive the Navier-Stokes equations together with explicit expressions for the fluxes and the transport coeffcients.

The hydrodynamic equations can be obtained in explicit form by taking the inner product of (2.2) with the hydrodynamic variables $\psi_{a}$, yielding equations of the form

$$
\frac{\partial}{\partial t}\left(\psi_{a}, h\right)=-\sum_{b}\left(\psi_{a}, L \psi_{b}\right)\left(\psi_{b}, h\right)\left(\psi_{b}, \psi_{b}\right)^{-1}-\left(P_{\perp} L^{T} \psi_{a}, P_{\perp} h\right) .
$$

Since $P_{\perp} h$ has been expressed through (2.17) and (2.6) in terms of local hydrodynamic variables, eq. (3.1) is a closed set of hydrodynamic equations. For the case $a=l$ we obtain the equations of continuity, using (2.8), (1.10), (2.5) and $(2.16 \mathrm{~b})$, in the form

$$
\frac{\partial}{\partial t} n_{l}=-\mathrm{i} \boldsymbol{k} \cdot n_{l} \delta \boldsymbol{u}-\mathrm{i} \boldsymbol{k} \cdot \boldsymbol{j}_{l} / m_{l}
$$

where we have identified the mass $f u x \boldsymbol{j}_{l}$ of species $l$ as

$$
\dot{j}_{l}=\left(J_{l}, P_{\perp} h\right),
$$

with

$$
J_{i i}=m_{i} v_{i}\left(\delta_{i l}-\varrho_{i} / \varrho\right)
$$


For the total mass density $\delta \varrho=\sum_{l} m_{l} \delta n_{l}$ we have from (3.2)-(3.4),

$$
\frac{\partial}{\partial t} \delta \varrho=-i \boldsymbol{k} \cdot \varrho \delta \boldsymbol{u}
$$

For the case $a=u$ we obtain the equation of motion, using (2.8), (1.10), (2.5) and (2.16c),

$$
\varrho \frac{\partial}{\partial t} \delta u=-\mathrm{i} k \delta p-\mathrm{i} k \cdot \sigma
$$

where $\delta p$ is the linear deviation of the local pressure from its equilibrium value which is given by

$$
\delta p=\beta^{-1} \sum_{j, l} n_{j} \Delta_{j l} \frac{\delta n_{l}}{n_{l}}+p \frac{\delta T}{T} .
$$

In order to prove the validity of (3.7) we use the identity, which has been derived in paper I

$$
\Delta_{j l}=\delta_{j l}-n_{l} \tilde{C}_{J l}(0)=\beta n_{l}\left(\frac{\partial \mu_{j}}{\partial n_{l}}\right)_{T, n_{i \neq l}},
$$

where $\mu_{j}$ is the chemical potential of species $j$. In taking the derivative we keep the temperature and all partial densities, except $n_{l}$, constant. Insertion of (3.8) into (3.7) yields the Gibbs-Duhem relation

$$
\delta p=\sum_{j l} n_{j}\left(\frac{\partial \mu_{j}}{\partial n_{l}}\right)_{T, n_{l \neq l}} \delta n_{l}+\frac{p}{T} \delta T=\sum_{j} n_{j} \delta \mu_{j}+s \delta T,
$$

where we have used that the deviation $\delta \mu_{j}$ is given by

$$
\delta \mu_{j}=\sum_{l}\left(\frac{\partial \mu_{j}}{\partial n_{l}}\right)_{T, n_{i \neq l}} \delta n_{l}+\left(\frac{\partial \mu_{j}}{\partial T}\right)_{n_{i}} \delta T
$$

and that $(P / T)-\sum n_{j}\left(\partial \mu_{j} / \partial T\right)_{n_{t}}=(\partial f / \partial T)_{n_{t}}=s$, where $f$ is the free-energy density, $s$ is the entropy density and the property $p / T=(\partial p / \partial T)_{n_{l}}$, valid for a hardsphere system, has been used.

In (3.6) we have further identified the momentum flux or pressure tensor $\sigma$ (actually its longitudinal components $\hat{\boldsymbol{k}} \cdot \boldsymbol{\sigma}$, which are the only relevant parts of $\boldsymbol{\sigma}$ as far as the hydrodynamic equations are concerned)

$$
\hat{\boldsymbol{k}} \cdot \boldsymbol{\sigma}=\hat{\boldsymbol{k}} \cdot\left(\boldsymbol{\Sigma}, P_{\perp} h\right)-\eta^{\prime} \mathrm{i} k(\delta \boldsymbol{u}+2 \hat{\boldsymbol{k}} \hat{\boldsymbol{k}} \cdot \delta \boldsymbol{u}),
$$


with

$$
\begin{aligned}
& \mathbf{\Sigma}=\Pi+1 \Gamma, \\
& \Pi_{i}=\left\{m_{i} v_{i} v_{i}\right\}_{\mathrm{s}} A_{i}, \\
& \Gamma_{i}=\left(\frac{1}{3} m_{i} v_{i}^{2}-k_{\mathrm{B}} T\right) \sum_{j}\left(\delta_{i j}-n_{J} / n\right) B_{j} .
\end{aligned}
$$

For the case $a=T$ we find from (2.8), (1.10), (2.5) and (2.16d) the rate of change of the local temperature,

$$
\frac{3}{2} n k_{\mathrm{B}} \frac{\partial}{\partial t} \delta T=-\mathrm{i} \boldsymbol{k} \cdot p \delta \boldsymbol{u}-\mathrm{i} \boldsymbol{k} \cdot \boldsymbol{j}_{q}+\mathrm{i} \boldsymbol{k} \cdot \sum_{l} \frac{3 k_{\mathrm{B}} T}{2 m_{l}} \boldsymbol{j}_{l},
$$

where the energy flux has been identified as

$$
j_{q}=\left(J_{q}, P_{\perp} h\right)-\lambda^{\prime} \mathrm{i} k(\delta T / T),
$$

with

$$
J_{q_{i}}=v_{i}\left(\frac{1}{2} m_{i} v_{i}^{2}-\frac{5}{2} k_{\mathrm{B}} T\right) E_{l}+\sum_{l}\left(k_{\mathrm{B}} T / m_{l}\right)\left(F_{l}+\frac{3}{2}\right) \boldsymbol{J}_{l i}
$$

The reason for identifying $\boldsymbol{j}_{\boldsymbol{q}}$ as the energy flux becomes clear if one considers the equation for the energy balance. Since for a hard-sphere system the deviation $\delta e$ of the local energy density from its equilibrium value is given by

$$
\delta e=\frac{3}{2} n k_{\mathrm{B}} \delta T+\frac{3}{2} k_{\mathrm{B}} T \sum_{l} \delta n_{l},
$$

the energy balance equation can be obtained directly from (3.2) and (3.13) in the standard form

$$
\frac{\partial}{\partial t} \delta e=-\mathrm{i} k \cdot\left(\frac{3}{2} n k_{\mathrm{B}} T+p\right) \delta u-\mathrm{i} k \cdot j_{q},
$$

where $\frac{3}{2} n k_{\mathrm{B}} T+p$ is the enthalpy density of the hard-sphere mixture.

The next part of this section deals with the fluxes and corresponding transport coefficients. As can be seen from the definitions of the fluxes and the expression for $P_{\perp} h$, derived in the previous section, the fluxes are proportional to the gradients of the partial densities, of the mass velocity, and of the temperature, which act as driving forces for these fluxes. The coefficients of proportionality are linear transport coefficients for which we are seeking explicit expressions. The theory of irreversible thermodynamics tells us that we have to choose the fluxes and thermodynamic driving forces in conjugate pairs, such that the irreversible entropy 
production $\sigma_{\mathrm{irr}}$ can be written in the form $\sigma_{\mathrm{irr}} T=\sum_{i} j_{i} X_{i}$, where the summation runs over all pairs of conjugate fluxes and forces. Here we make the same choice of fluxes $j$ and conjugate driving forces $X$ as in paper I. For reasons of convenience the Fourier transforms of the forces are given,

$$
\begin{aligned}
& \boldsymbol{j}_{0}=\boldsymbol{j}_{q}-\sum_{l} \frac{1}{m_{l}}\left(\frac{\partial e}{\partial n_{l}}\right)_{T, n_{l \neq l}} \quad \boldsymbol{j}_{l}=\boldsymbol{j}_{q}-\sum_{l} \frac{3 k_{\mathrm{B}} T}{2 m_{l}} j_{l} ; \quad \boldsymbol{X}_{0}=-\mathrm{i} \boldsymbol{k} \frac{\delta T}{T}, \\
& \boldsymbol{j}_{l} ; \quad \boldsymbol{X}_{l}=-\mathrm{i} \boldsymbol{k} \frac{\left(\delta \mu_{l}\right)_{T}}{m_{l}}=-\mathrm{i} k \frac{k_{\mathrm{B}} T}{m_{l}} \sum_{j} \Delta_{l j} \frac{\delta n_{j}}{n_{j}} \\
& \boldsymbol{\sigma} ; \quad \mathbf{X}_{\boldsymbol{u}}=-\mathrm{i} \boldsymbol{k} \delta \boldsymbol{u} .
\end{aligned}
$$

Here $\mathrm{i} k\left(\delta \mu_{l}\right)_{T}$ is the (Fourier transform of the) gradient of $\mu_{l}$ at constant temperature. The second expressions for $\boldsymbol{j}_{0}$ and $\boldsymbol{X}_{l}$ are specific for hard spheres, all the other expressions apply equally well to more general systems. This choice for the conjugate sets of fluxes and forces is particularly convenient for hard-sphere systems, since the rate equation for the temperature reduces to the simple form

$$
\frac{3}{2} n k_{\mathrm{B}} \frac{\partial}{\partial t} \delta T=-\mathrm{i} \boldsymbol{k} \cdot \delta \boldsymbol{u} p-\mathrm{i} \boldsymbol{k} \cdot \boldsymbol{j}_{0} .
$$

In order to introduce the forces $\boldsymbol{X}_{0}, \boldsymbol{X}_{l}$ and $\mathbf{X}_{u}$ in $P_{\perp} h$, given by (2.17) we have to eliminate the gradients of the partial densities in favour of the diffusion forxes $X_{l}$. In order to do so we write down the explicit form of $P_{1} h$ by collecting the results from (2.17), (2.6), (2.16), (3.4), (3.12), (3.15) and (3.18), yielding

$$
P_{\perp} h=-\Lambda^{-1}(0)\left(\mathrm{i} k \cdot \sum_{j, l} \frac{J_{j}}{m_{j}} \Delta_{J l} \frac{\delta n_{l}}{n_{l}}+\beta \mathbf{\Sigma}: \mathbf{X}_{u}+\beta J_{q} \cdot \boldsymbol{X}_{0}-\sum_{l} \frac{3}{2 m l} J_{l} \cdot \boldsymbol{X}_{0}\right),
$$

or

$$
P_{\perp} h=\beta \Lambda^{-1}(0)\left(J_{0} \cdot X_{0}+\sum_{l} J_{l} \cdot X_{l}+\Sigma: \mathbf{X}_{u}\right),
$$

where we introduced the microscopic heat flux

$$
J_{0}=J_{q}-\sum_{l} \frac{3}{2}\left(k_{\mathrm{B}} T / m_{l}\right) J_{l} .
$$

As we have derived expressions for the fluxes and for the function $P_{\perp} h$, we can directly calculate the flux $j_{0}$ and the mass fluxes $\boldsymbol{j}_{l}$, which follow from (3.18a), (3.14), (3.3) and (3.20) to be

$$
\begin{aligned}
& \boldsymbol{j}_{0}=\beta\left(\boldsymbol{J}_{0}, \Lambda^{-1}(0) J_{0}\right) \cdot \boldsymbol{X}_{0}+\beta \sum_{\boldsymbol{l}}\left(\boldsymbol{J}_{0}, \Lambda^{-1}(0) \boldsymbol{J}_{l}\right) \cdot \boldsymbol{X}_{\boldsymbol{l}}-\lambda^{\prime} \boldsymbol{X}_{0}, \\
& \boldsymbol{j}_{l}=\beta\left(\boldsymbol{J}_{l}, \Lambda^{-1}(0) \boldsymbol{J}_{0}\right) \cdot \boldsymbol{X}_{0}+\beta \sum_{l^{\prime}}\left(\boldsymbol{J}_{l}, \Lambda^{-1}(0) \boldsymbol{J}_{l^{\prime}}\right) \cdot \boldsymbol{X}_{l^{\prime}},
\end{aligned}
$$


where we have used that isotropic tensors of the form $\left(J, \Lambda^{-1}(0) \Sigma\right)$ vanish, i.e., fluxes couple only to the driving forces of the same tensorial character. This property is known in irreversible thermodynamics as Curie's law ${ }^{11}$ ). Since the second-rank tensors in (3.22) are isotropic, they are a multiple of the unit tensor 1 , and (3.22) can be written as

$$
j_{0}=L_{00} X_{0}+\sum_{l} L_{0 l} X_{l}, \quad j_{l}=L_{l 0} X_{0}+\sum_{l^{\prime}} L_{l l^{\prime}} \boldsymbol{X}_{l^{\prime}},
$$

where the coefficients $L_{00}, L_{0 l}, L_{l 0}$ and $L_{l l}$, are linear transport coefficients which can be expressed as a linear combination of standard transport coefficients, like the heat-conductivity coefficient, the Dufour coefficients, the thermodiffusion or Soret coefficients and the multicomponent diffusion coefficients. Our coefficients are given by the following expressions;

$$
\begin{aligned}
& L_{00}=\frac{1}{3} \beta\left(J_{0}^{\alpha}, \Lambda^{-1}(0) J_{0}^{\alpha}\right)+\lambda^{\prime}, \\
& L_{0 l}=\frac{1}{3} \beta\left(J_{0}^{\alpha}, \Lambda^{-1}(0) J_{l}^{\alpha}\right), \\
& L_{l 0}=\frac{1}{3} \beta\left(J_{l}^{\alpha}, \Lambda^{-1}(0) J_{0}^{\alpha}\right), \\
& L_{l l^{\prime}}=\frac{1}{3} \beta\left(J_{l}^{\alpha}, \Lambda^{-1}(0) J_{l^{\prime}}^{\alpha}\right) .
\end{aligned}
$$

The microscopic fluxes are given explicitly in (3.4), (3.21) and (3.15). The constants $E_{i}$ and $F_{i}$ appearing in (3.15) are listed in (2.14) and $\lambda^{\prime}$ is defined in (2.11b). In (3.24) the summation convention for repeated indices is used. Notice the Onsager symmetry relations

$$
\begin{aligned}
& L_{l l^{\prime}}=L_{l^{\prime} l}, \\
& L_{0 l}=L_{l 0},
\end{aligned}
$$

which are implied by the symmetry of the operator $A(0)$, [see (1.16)]. In addition the matrix of transport coefficients $L_{i j}(i, j=0,1,2, \ldots, s)$ is positive definite as follows from (1.17) and (1.18), since the microscopic fluxes $J_{i}$ in (3.24) are orthogonal to the null eigenfunctions of $\Lambda(0)$.

Finally we consider the pressure tensor, which can be deduced from (3.11), (3.20) and (3.18c)

$$
\hat{\boldsymbol{k}} \cdot \boldsymbol{\sigma}=\hat{\boldsymbol{k}} \beta \cdot\left(\Sigma, \Lambda^{-1}(0) \Sigma\right): \mathbf{X}_{u}+\eta^{\prime}\left(\hat{\boldsymbol{k}} \cdot \mathbf{X}_{u}+2 \mathbf{X}_{u} \cdot \hat{\boldsymbol{k}}\right) .
$$

Again we have used Curie's law. From the isotropy of the fourth-rank tensor $\left(\Sigma^{\alpha \beta}, A^{-1}(0) \Sigma^{\gamma \delta}\right)$, which is symmetric in the labels $(\alpha, \beta)$ and in the labels $(\gamma, \delta)$ [see (3.12)], follows that it contains only two independent constants, so that

$$
\beta\left(\Sigma^{\alpha \beta}, \Lambda^{-1}(0) \Sigma^{\gamma \delta}\right)=\eta^{\prime \prime}\left(\delta_{\alpha \gamma} \delta_{\beta \delta}+\delta_{\alpha \delta} \delta_{\beta \gamma}-\frac{2}{3} \delta_{\alpha \beta} \delta_{\gamma \delta}\right)+\zeta^{\prime \prime} \delta_{\alpha \beta} \delta_{\gamma \tilde{\delta}} .
$$


By making appropriate contractions in (3.27) one obtains

$$
\begin{aligned}
\eta^{\prime \prime} & =\frac{1}{10} \beta\left(\Pi^{\alpha \beta}, \Lambda^{-1}(0) \Pi^{\alpha \beta}\right), \\
\zeta^{\prime \prime} & =\frac{1}{9} \beta\left(\Sigma^{\alpha \alpha}, \Lambda^{-1}(0) \Sigma^{\beta \beta}\right)=\beta\left(\Gamma, \Lambda^{-1}(0) \Gamma\right),
\end{aligned}
$$

where $\Pi^{\alpha \beta}$ and $\Gamma$ are defined in (3.12). Insertion of (3.27) in (3.26) yields

$$
\hat{\boldsymbol{k}} \cdot \boldsymbol{\sigma}=-\eta\left(\hat{\boldsymbol{k}} \cdot \mathbf{X}_{u}+\frac{1}{3} \mathbf{X}_{u} \cdot \hat{\boldsymbol{k}}\right)+\zeta \mathbf{X}_{u} \cdot \hat{\boldsymbol{k}},
$$

where $\eta$ is the shear viscosity and $\zeta$ the bulk viscosity, given by

$$
\eta=\eta^{\prime}+\eta^{\prime \prime} ; \quad \zeta=\zeta^{\prime \prime}+\frac{5}{3} \eta^{\prime} .
$$

The quantities $\eta^{\prime}, \eta^{\prime \prime}$ and $\zeta^{\prime \prime}$ are defined in (2.11a) and (3.32). The fluxes $\Pi$ and $\Gamma$, listed in (3.9), contain the constants $A_{i}$ and $B_{i}$ defined in (2.14). Both viscosities are again positive, due to the positive-definiteness of the operator $A(0)$.

4. Conclusion. By linearizing the modified Enskog equation, defined in paper I, we obtained the Navier-Stokes equations for a mixture of hard spheres, together with explicit expressions for the linear transport coefficients. The form of these equations turned out to be in complete agreement with the phenomenological laws of irreversible thermodynamics. The matrix of transport coefficient satisfies the Onsager reciprocity relations and is positive definite. Using this result we have shown in I that the existing extensions of the Enskog equation to the case of hardsphere mixtures ${ }^{3,4,5}$ ) in general do not satisfy the laws of irreversible thermodynamics; more specifically the Onsager relations are not satisfied.

The results for the single component case can be read off directly from our results. For the bulk viscosity one finds directly from (3.30), (3.28), (3.11) and (2.11a) that

$$
\zeta=\frac{4}{9} n^{2} \chi \sigma^{4}(\pi m k T)^{\frac{1}{2}} \equiv \bar{\omega},
$$

where $\bar{\omega}$ is the symbol used by Chapman and Cowling. For the shear viscosity and heat-conduction coefficient one obtains

$$
\begin{aligned}
& \eta=\frac{1}{\chi}\left(1+\frac{4}{15} n \chi \pi \sigma^{3}\right)^{2} \eta^{(0)}+\frac{3}{5} \bar{\omega}, \\
& \lambda=\frac{L_{00}}{T}=\frac{1}{\chi}\left(1+\frac{2}{3} n \chi \pi \sigma^{3}\right)^{2} \lambda^{(0)}+C_{v} \bar{\omega},
\end{aligned}
$$

where $C_{v}=3 k_{\mathrm{B}} / 2 m$ and $\eta^{(0)}$ and $\lambda^{(0)}$ are the Boltzmann values (low-density limit) of $\eta$ and $\lambda$. 
The results for the mixtures, however, cannot be related in such a simple way to their low-density limits. For the diffusion coefficient in a binary mixture one deduces directly from (3.24) that in first Enskog approximation

$$
\left[L_{12}\right],=\left(1 / \chi_{12}\right)\left[L_{12}^{(0)}\right],
$$

where [...], indicates the first Enskog approximation, and $L_{12}^{(0)}$ the Boltzmann value of the diffusion coefficient. In ref. 2 a simple method is given for relating the Enskog result for mixtures to the Boltzmann result, which can be applied here too.

The explicit expressions for the transport coefficients, derived in section 3, can be evaluated directly in successive Enskog approximations by using the standard method, given in ref. 3 .

For a more general discussion and comparison between our extension of Enskog's theory to mixtures and the old versions we refer to paper I.

\section{APPENDIX}

The matrix $\left(\psi_{a}, L \psi_{b}\right)$ can be calculated from (1.2), (1.8) and (1.9), where $L=K+D+A$. Trivial calculations give

$$
\left(\psi_{a}, K \psi_{b}\right)=\left(\begin{array}{ccc}
0 & \mathrm{i} k \beta^{-1} n_{l} & 0 \\
\mathrm{i} k \beta^{-1} n_{l} & 0 & \mathrm{i} k \beta^{-2} n \\
0 & \mathrm{i} k \beta^{-2} n & 0
\end{array}\right)
$$

and

$$
\left(\psi_{a}, D \psi_{b}\right)=\left(\begin{array}{ccc}
0 & 0 & 0 \\
-\mathrm{i} k \beta^{-1} \sum_{i} n_{l} n_{l}\left[\tilde{C}_{u l}(k)-\chi_{u l} \tilde{f}_{i l}(k)\right] & 0 & 0 \\
0 & 0 & 0
\end{array}\right) .
$$

The meaning of row and column labels is given below (2.8). In order to obtain $\left(\psi_{a}, \Lambda \psi_{b}\right)$ we employ (1.14). First observe that $\Lambda^{T} \chi_{l}=0$, as can be seen from (1.12c); consequently

$$
\left(\psi_{a}, \Lambda^{T} \psi_{l}\right)=\left(w_{l}, \Lambda \psi_{a}\right)=0 .
$$

Similarly one finds from (A.3), (1.14) and $\Delta \psi_{T}=\Delta^{T} \psi_{T}=0$

$$
\begin{aligned}
& \left(\psi_{u}, A \psi_{l}\right)=\left(\psi_{u}, \Delta \psi_{l}\right)=-\mathrm{i} k \beta^{-1} \sum_{t} n_{i} n_{l} \chi_{l l} f_{l l}(k), \\
& \left(\psi_{T}, A \psi_{l}\right)=\left(\psi_{T}, \Delta \psi_{l}\right)=0 \\
& \left(\psi_{u}, A \psi_{u}\right)=\left(\psi_{u}, \Lambda^{\top} \psi_{u}\right)
\end{aligned}
$$




$$
\begin{aligned}
& \left(\psi_{u}, \Lambda \psi_{T}\right)=\left(\psi_{u}, \Lambda^{T} \psi_{T}\right)=\left(\psi_{T}, \Lambda \psi_{u}\right), \\
& \left(\psi_{T}, \Lambda \psi_{T}\right)=\left(\psi_{T}, \Lambda^{T} \psi_{T}\right) .
\end{aligned}
$$

The matrix elements (A.4c)-(A.4e) remain to be calculated. Consider first (A.4e). By virtue of $(1.12 \mathrm{c})$ we can write

$$
\begin{aligned}
\left(\psi_{T}, A \psi_{T}\right)= & -\sum_{i j} \chi_{i j} \sigma_{i j}^{2} \int \mathrm{d} v_{i} \mathrm{~d} v_{j} \varphi_{t} \varphi_{j} \int^{\prime} \mathrm{d} \hat{\sigma}\left(\boldsymbol{v}_{i j} \cdot \hat{\sigma}\right) \\
& \times\left(\mathrm{e}^{-i k \cdot \hat{\sigma} \sigma_{i j}}-1\right)\left(\frac{1}{2} m_{i} v_{i}^{2}-\frac{3}{2} \beta^{-1}\right) \frac{1}{2} m_{j}\left(v_{j}^{* 2}-v_{j}^{2}\right) .
\end{aligned}
$$

We use the dynamics (1.4) and change to centre of mass and relative velocities,

$$
\boldsymbol{v}_{i}=G_{i j}+\left(\mu_{i j} / m_{i}\right) v_{i j} ; \quad v_{j}=G_{i j}-\left(\mu_{i j} / m_{j}\right) v_{i j} .
$$

The jacobian of this transformation is 1 , and we write $\varphi_{t} \varphi_{j}=n_{t} n_{J} \varphi_{\mathrm{c}} \varphi_{\mathrm{r}}$, with

$$
\begin{aligned}
& \varphi_{\mathrm{c}}=\varphi_{\mathrm{c}}(G)=\left(\beta M_{i j} / 2 \pi\right)^{3 / 2} \exp \left(-\frac{1}{2} \beta M_{i j} G^{2}\right), \\
& \varphi_{\mathrm{r}}=\varphi_{\mathrm{r}}(v)=\left(\beta \mu_{i j} / 2 \pi\right)^{3 / 2} \exp \left(-\frac{1}{2} \beta \mu_{i j} v^{2}\right),
\end{aligned}
$$

where $M_{i j}=m_{i}+m_{j}$ and $\mu_{i j}^{-1}=m_{i}^{-1}+m_{j}^{-1}$. Next, we carry out the integration over the centre of mass velocity $\boldsymbol{G}_{i j}$, yielding

$$
\begin{aligned}
\left(\psi_{T}, A \psi_{T}\right)= & -\beta^{-1} \sum_{i j}\left(2 \mu_{i j}^{2} / M_{i j}\right) n_{i} n_{j} \chi_{i j} \sigma_{i j}^{2} \\
& \times \int \mathrm{d} v \varphi_{i j} \int_{i j}^{\prime} \mathrm{d} \hat{\sigma}(v \cdot \hat{\sigma})^{3} \cdot\left(\mathrm{e}^{-\mathrm{i} k \cdot \hat{\sigma} \sigma_{i j}}-1\right) .
\end{aligned}
$$

After changing the order of integrations, we can carry out the $v$ integration,

$$
\int_{v \cdot \hat{\sigma}>0} \mathrm{~d} v(v \cdot \hat{\boldsymbol{\sigma}})^{3} \varphi_{\mathrm{r}}=(2 / \pi)^{\frac{1}{2}}(\beta \mu)^{-3 / 2} .
$$

The $\hat{\sigma}$ integration can be expressed in terms of spherical Bessel functions $j_{l}(\varrho)$ $(l=0,1,2, \ldots)$, defined as

$$
\begin{aligned}
& j_{0}(\varrho)=\frac{\sin \varrho}{\varrho} ; \quad j_{1}(\varrho)=\frac{\sin \varrho}{\varrho^{2}}-\frac{\cos \varrho}{\varrho}, \\
& j_{2}(\varrho)=\left(\frac{3}{\varrho^{3}}-\frac{1}{\varrho}\right) \sin \varrho-\frac{3}{\varrho^{2}} \cos \varrho,
\end{aligned}
$$

which satisfy the recursion relation

$$
\frac{\mathrm{d}}{\mathrm{d} \varrho} j_{l}=\frac{1}{2 l+1}\left[l j_{l-1}-(l+1) j_{l+1}\right] \text {. }
$$


By virtue of (A.6) we can write in (A.5)

$$
\int \mathrm{d} \hat{\sigma}\left(\mathrm{e}^{-\mathrm{i} k \cdot \hat{\sigma} \sigma_{I J}}-1\right)=4 \pi\left[j_{0}\left(k \sigma_{i j}\right)-1\right] .
$$

Collecting the above results then gives

$$
\left(\psi_{T}, \Lambda \psi_{T}\right)=\beta^{-1} k^{2} \lambda^{\prime}(k),
$$

where

$$
k^{2} \lambda^{\prime}(k)=8 \beta^{-1} \sum_{i j} n_{i} n_{j} \sigma_{i j}^{2} \chi_{i j}\left[1-j_{0}\left(k \sigma_{i j}\right)\right]\left(2 \pi \mu_{i j} / \beta\right)^{\frac{1}{2}} M_{i j}^{-1} .
$$

In a similar way we find for (A.4c)

$$
\left(\psi_{u}, A \psi_{u}\right)=\beta^{-1} k^{2}\left[\eta^{\prime}(k)(1-\hat{k} \hat{k})+\eta^{\prime \prime}(k) \hat{k} \hat{k}\right],
$$

where

$$
\begin{aligned}
& k^{2} \eta^{\prime}(k)=\frac{8}{3} \sum_{i j} n_{t} n_{j} \chi_{i j} \sigma_{i j}^{2}\left(2 \pi \mu_{i j} / \beta\right)^{\frac{1}{2}}\left[1-j_{0}\left(k \sigma_{i j}\right)-j_{2}\left(k \sigma_{i j}\right)\right], \\
& k^{2} \eta^{\prime \prime}(k)=\frac{8}{3} \sum_{i j} n_{t} n_{j} \chi_{i j} \sigma_{t j}^{2}\left(2 \pi \mu_{t j} / \beta\right)^{\frac{1}{2}}\left[1-j_{0}\left(k \sigma_{i j}\right)+2 j_{2}\left(k \sigma_{i j}\right)\right] .
\end{aligned}
$$

In the derivation of (A.10) we have used the following relation for an isotropic tensor field

$$
\int \mathrm{d} \hat{\boldsymbol{\sigma}} \mathrm{e}^{-\mathrm{i} \varrho \hat{\boldsymbol{k}} \cdot \hat{\sigma}} \hat{\boldsymbol{\sigma}} \hat{\sigma}=a(\varrho)(1-\hat{\boldsymbol{k}} \hat{\boldsymbol{k}})+b(\varrho) \hat{\boldsymbol{k}} \hat{\boldsymbol{k}},
$$

where the coefficients $a$ and $b$ can be determined by contraction, i.e.,

$$
\begin{aligned}
& 2 a+b=\int \mathrm{d} \hat{\sigma} \mathrm{e}^{-\mathrm{i} \mathrm{e} \hat{k} \cdot \hat{\sigma}}=4 \pi j_{0}(\varrho), \\
& b=\int \mathrm{d} \hat{\sigma} \mathrm{e}^{-\mathrm{i} \hat{k} \cdot \hat{\sigma}}(\hat{\boldsymbol{\kappa}} \cdot \hat{\sigma})^{2}=\frac{4}{3} \pi\left[j_{0}(\varrho)-2 j_{2}(\varrho)\right]
\end{aligned}
$$

and where (A.7) has been used. The remaining matrix element (A.4d) is calculated again in a similar way and yields

$$
\left(\psi_{u}, \Lambda \psi_{u}\right)=-\mathrm{i} k \beta^{-2} \frac{1}{2} \sum_{i j} n_{i} n_{j} \chi_{i j} f_{i j}(k) .
$$

Addition of the matrix elements in (A.1)-(A.5), (A.10) and (A.12) yields finally

$$
\begin{aligned}
& \left(\psi_{a}, L \psi_{b}\right) \\
& \quad=\left(\begin{array}{ccc}
0 & \mathrm{i} k \beta^{-1} n_{l} & \text { (A.13) } \\
\mathrm{i} k \beta^{-1} \sum n_{i} \Delta_{t l}(k) & k^{2} \beta^{-1}\left[\eta^{\prime}(k)(1-\hat{\boldsymbol{k}} \hat{k})+\eta^{\prime \prime}(k) \hat{k} \hat{k}\right] & \mathrm{i} k \beta^{-1} p(k) \\
0 & \mathrm{i} k \beta^{-1} p(k) & k^{2} \beta^{-1} \lambda^{\prime}(k)
\end{array}\right),
\end{aligned}
$$


where

$$
\Delta_{i l}(k)=\delta_{i l}-n_{l} \widetilde{C}_{i l}(k), \quad \beta p(k)=n-\frac{1}{2} \sum_{i j} n_{i} n_{j} \chi_{i j} \tilde{f}_{i j}(k) .
$$

The quantity $p(k)$ reduces in the long-wavelength limit to the equilibrium pressure of a hard-sphere mixture.

In the body of the paper the matrix $\left(\psi_{a}, L \psi_{b}\right)$ is only needed to terms of $\mathcal{O}\left(k^{2}\right)$. Therefore we make a $k$ expansion of the matrix elements in ( $\Lambda .11)$. Since the functions $\Delta_{i j}(k), f_{i j}(k), \eta^{\prime}(k), \zeta^{\prime}(k)$ and $\lambda^{\prime}(k)$ contain only even powers of $k$, we find to the relevant order in $k$

$$
\left(\psi_{a}, L \psi_{b}\right)=\left(\begin{array}{ccc}
0 & \mathrm{i} k \beta^{-1} n_{l} & 0 \\
\mathrm{i} k \beta^{-1} \sum_{i} n_{l} \Delta_{l l} & k^{2} \beta^{-1} \eta^{\prime}(1+2 \hat{k} \hat{k}) & \mathrm{i} k \beta^{-1} p \\
0 & \mathrm{i} k \beta^{-1} p & k^{2} \beta^{-1} \lambda^{\prime}
\end{array}\right)
$$

which is eq. (2.8). The symbols are defined in (2.9), (2.10) and (2.11).

\section{REFERENCES}

1) Van Beijeren, H. and Ernst, M.H., Physica 68 (1973) 437.

2) Barajas, L., Garcia-Colin, L.S. and Pina, B., J. Stat. Phys. 7 (1973) 161.

3) Chapman, S. and Cowling, T. G., The Mathematical Theory of Nonuniform Gases, University Press (Cambridge 1960) p. 292.

4) Tham, M. K. and Gubbins, K. E., J. chem. Phys. 55 (1971) 268.

5) Bennet, D. E. and Curtiss, C. F., J. chem. Phys. 51 (1969) 2811.

6) De Groot, S. R., Thermodynamics of Irreversible Processes, North-Holland Publ. Comp. (Amsterdam 1951).

7) Zwanzig, R., Boulder Lectures in Theoretical Physics, W. E. Brittin. ed., Interscience Publishers, (New York, 1960).

8) Ernst, M. H., Haines, L. K. and Dorfman, J. R., Rev. mod. Phys. 41 (1969) 296.

9) Ref. 3, p. 117.

10) Ernst, M. H., Amer. J. Phys. 38 (1970) 908.

11) Ref. 6, p. 77. 